\title{
Validation of components of local ties
}

Susanne Glaser ${ }^{1,2}$, M. Fritsche $e^{1,3}$, K. Sośnica ${ }^{4}$,

C. J. Rodríguez-Solano ${ }^{5}$, K. Wang ${ }^{6}$, R. Dach ${ }^{4}$, U. Hugentobler ${ }^{5}$, M. Rothacher ${ }^{6}$, R. Dietrich ${ }^{1}$

${ }^{1}$ Technische Universität Dresden, ${ }^{2}$ now at: Technische Universität Berlin, ${ }^{3}$ now at: GFZ German Research Centre for Geosciences,

${ }^{4}$ Universität Bern, ${ }^{5}$ Technische Universität München, ${ }^{6}$ ETH Zürich

Kirchberg, October 13, 2014

G F Z

Helmholtz Centre POTSDAM
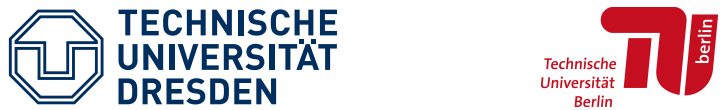


\section{Outline}

(1) Introduction 


\section{Outline}

(1) Introduction

(2) Single-technique solutions

- Input data

- Preliminary analysis

- Results 


\section{Outline}

(1) Introduction

(2) Single-technique solutions

- Input data

- Preliminary analysis

- Results

(3) Combined solution

- Strategy

- Results 


\section{Outline}

(1) Introduction

(2) Single-technique solutions

- Input data

- Preliminary analysis

- Results

(3) Combined solution

- Strategy

- Results

(4) Conclusion 


\title{
Outline
}

\author{
(1) Introduction
}

(2) Single-technique solutions

(3) Combined solution

(4) Conclusion 
Introduction

\section{Reference frame as the realization of a reference system}

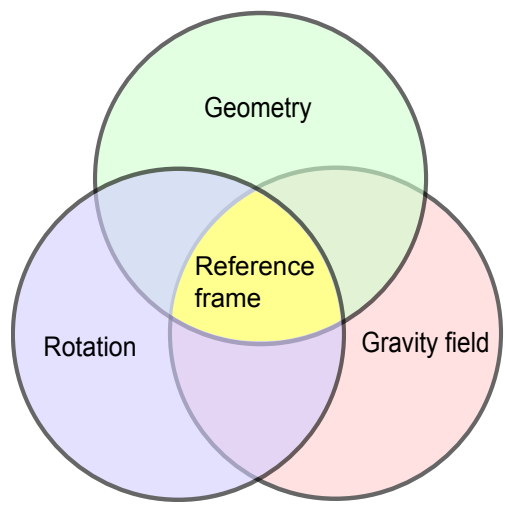

Figure: Reference frame as the connections of the three pillars of geodesy, according to IAG (2014) 
Introduction

\section{Combination of different geodetic space techniques}

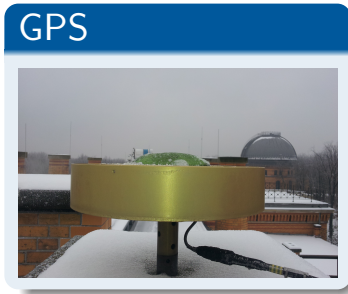

\section{DORIS}

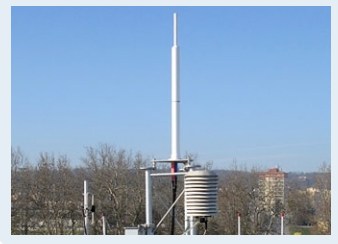

\section{SLR}

\section{$\mathrm{VLBI}$}
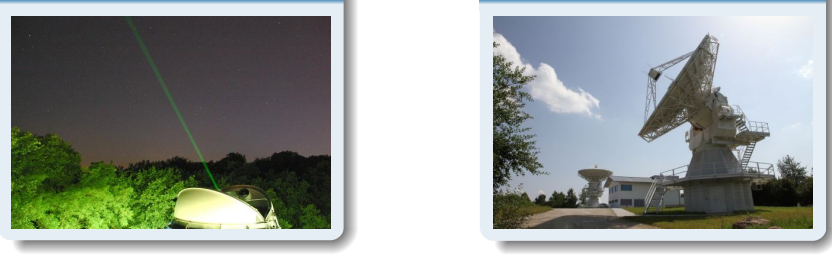
Motivation

\section{Combination strategy}

Combination of the different techniques 
Motivation

Combination strategy

Combination of the different techniques

- Local ties at co-located sites 
Motivation

\section{Combination strategy}

\section{Combination of the different techniques}

- Local ties at co-located sites

- Inhomogeneous data base

- IERS Working Group on Site Survey and Co-location 
Motivation

\section{Combination strategy}

\section{Combination of the different techniques}

- Local ties at co-located sites

- Inhomogeneous data base

- IERS Working Group on Site Survey and Co-location

- Pole coordinates as global ties (Seitz et al., 2012) 
Motivation

\section{Combination strategy}

\section{Combination of the different techniques}

- Local ties at co-located sites

- Inhomogeneous data base

- IERS Working Group on Site Survey and Co-location

- Pole coordinates as global ties (Seitz et al., 2012)

- Our approach: combination of the pole coordinates and the degree-1 surface load coefficients (Blewitt, 2003), common origin of GNSS and SLR 


\section{Outline}

(1) Introduction

(2) Single-technique solutions

(3) Combined solution

(4) Conclusion 


\section{Input data}

\section{Data}




\section{Data}

GNSS

- daily normal equation systems 1994 - 2010

- GPS and GLONASS

- 334 stations 
Input data

\section{Data}

GNSS

- daily normal equation systems 1994 - 2010

- GPS and GLONASS

- 334 stations
SLR

- weekly normal equation systems 1994 - 2010

- LAGEOS-1/-2

- 73 stations 


\section{Data}

GNSS

- daily normal equation systems 1994 - 2010

- GPS and GLONASS

- 334 stations
SLR
- weekly normal equation systems 1994 - 2010

- LAGEOS-1/-2

- 73 stations

\section{GPS, GLONASS and SLR}

from a homogeneous reprocessing (Fritsche et al., 2014) 


\section{Station network}

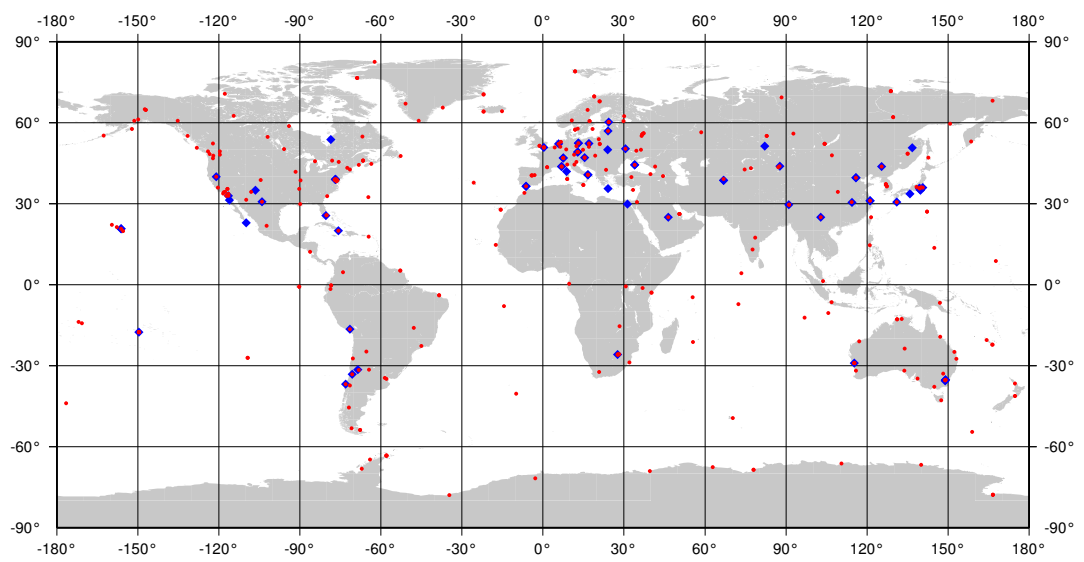

Figure: Globally distributed GNSS (red) and SLR (blue) stations 
Preliminary analysis at station positions time series of all stations 
Preliminary analysis at station positions time series of all stations

- Elimination of position outliers

- Station events (jumps)

- Core stations for the definition of the geodetic datum 
Preliminary analysis at station positions time series of all stations

- Elimination of position outliers

- Station events (jumps)

- Core stations for the definition of the geodetic datum

- Selection according to the length and accuracy of the station position time series and the global distribution

- Similar to IGS and ILRS solutions 
Results

\section{Pole coordinates}


Results

\section{Pole coordinates}
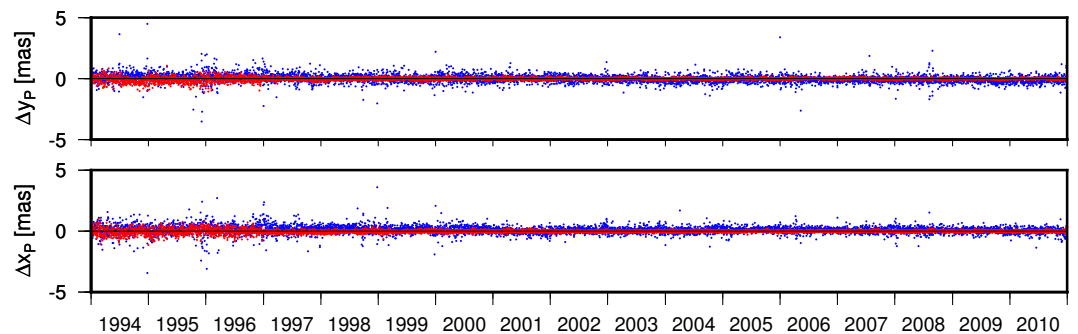

Figure: Differences $\Delta x_{P}, \Delta y_{P}$ of estimated and "IERS 08 C04" pole coordinates

\begin{tabular}{lllll}
\hline Offset, Trend & \multicolumn{2}{c}{ GNSS-only } & \multicolumn{2}{c}{ SLR-only } \\
\hline$\Delta x_{P}$ & $-0.04 \mathrm{mas}$ & $0.00 \mathrm{mas} / \mathrm{a}$ & $0.14 \mathrm{mas}$ & $0.00 \mathrm{mas} / \mathrm{a}$ \\
$\Delta y_{P}$ & $-0.06 \mathrm{mas}$ & $0.00 \mathrm{mas} / \mathrm{a}$ & $0.11 \mathrm{mas}$ & $0.00 \mathrm{mas} / \mathrm{a}$ \\
\hline
\end{tabular}




\section{Outline}

(1) Introduction

(2) Single-technique solutions

(3) Combined solution

(4) Conclusion 
Strategy

\section{Combination strategy and weighting}


Strategy

\section{Combination strategy and weighting}

$$
\mathbf{N} \cdot \hat{\mathbf{x}}=\mathbf{n}
$$




\section{Combination strategy and weighting}

$$
\mathbf{N} \cdot \hat{\mathbf{x}}=\mathbf{n}
$$

with

$$
\begin{aligned}
\mathbf{N} & =w_{G N S S} \mathbf{N}_{G N S S}+w_{S L R} \mathbf{N}_{S L R} \\
\mathbf{n} & =w_{G N S S} \mathbf{n}_{G N S S}+w_{S L R} \mathbf{n}_{S L R}
\end{aligned}
$$




\section{Combination strategy and weighting}

$$
\mathbf{N} \cdot \hat{\mathbf{x}}=\mathbf{n}
$$

with

$$
\begin{aligned}
\mathbf{N} & =w_{G N S S} \mathbf{N}_{G N S S}+w_{S L R} \mathbf{N}_{S L R} \\
\mathbf{n} & =w_{G N S S} \mathbf{n}_{G N S S}+w_{S L R} \mathbf{n}_{S L R}
\end{aligned}
$$

and

$$
\begin{aligned}
w_{G N S S} & =1 \\
w_{S L R} & =\frac{s_{G N S S}^{2}}{s_{S L R}^{2}}
\end{aligned}
$$




\section{Strategy}

\section{Combination strategy and weighting}

$$
\mathbf{N} \cdot \hat{\mathbf{x}}=\mathbf{n}
$$

with

$$
\begin{aligned}
\mathbf{N} & =w_{G N S S} \mathbf{N}_{G N S S}+w_{S L R} \mathbf{N}_{S L R} \\
\mathbf{n} & =w_{G N S S} \mathbf{n}_{G N S S}+w_{S L R} \mathbf{n}_{S L R}
\end{aligned}
$$

and

$$
\begin{aligned}
w_{G N S S} & =1 \\
w_{S L R} & =\frac{s_{G N S S}^{2}}{s_{S L R}^{2}} \cdot \frac{N_{G N S S}^{\text {mean }}}{N_{S L R}^{\text {mean }}}=0.81
\end{aligned}
$$




\section{Definition of the geodetic datum}

\section{Combination strategy}

- Combination of the pole coordinates of GNSS and SLR

- Combination of the degree-1 surface load coefficients

- Station velocities at co-located sites were set to be equal 


\section{Definition of the geodetic datum}

\section{Combination strategy}

- Combination of the pole coordinates of GNSS and SLR

- Combination of the degree-1 surface load coefficients

- Station velocities at co-located sites were set to be equal 


\section{Definition of the geodetic datum}

\section{Combination strategy}

- Combination of the pole coordinates of GNSS and SLR

- Combination of the degree-1 surface load coefficients

- Station velocities at co-located sites were set to be equal

Realization of the geodetic datum

- Origin: GNSS and SLR observations 


\section{Definition of the geodetic datum}

\section{Combination strategy}

- Combination of the pole coordinates of GNSS and SLR

- Combination of the degree-1 surface load coefficients

- Station velocities at co-located sites were set to be equal

Realization of the geodetic datum

- Origin: GNSS and SLR observations

- Network scale: GNSS and SLR observations 


\section{Definition of the geodetic datum}

\section{Combination strategy}

- Combination of the pole coordinates of GNSS and SLR

- Combination of the degree-1 surface load coefficients

- Station velocities at co-located sites were set to be equal

Realization of the geodetic datum

- Origin: GNSS and SLR observations

- Network scale: GNSS and SLR observations

- Orientation:

- NNR around the $X, Y, Z$ axis for GNSS

- NNR around the $Z$ axis for SLR

temporal change: NNR around the $X, Y, Z$ axis for GNSS 


\section{Definition of the geodetic datum}

\section{Combination strategy}

- Combination of the pole coordinates of GNSS and SLR

- Combination of the degree-1 surface load coefficients

- Station velocities at co-located sites were set to be equal

Realization of the geodetic datum

- Origin: GNSS and SLR observations

- Network scale: GNSS and SLR observations

- Orientation:

- NNR around the $X, Y, Z$ axis for GNSS

- NNR around the $Z$ axis for SLR

temporal change: NNR around the $X, Y, Z$ axis for GNSS

$\rightarrow$ minimum constraint solution 
Results

Estimation of components of the local ties 
Results

\section{Estimation of components of the local ties}

\section{Strategy}

- a priori positions and velocities

- $\Delta \mathbf{X}^{L T}=\mathbf{X}_{S L R}^{L T}-\mathbf{X}_{G N S S}^{L T}$

- same velocities for the LT stations 
Results

Estimation of components of the local ties

\section{Strategy}

- a priori positions and velocities

- $\Delta \mathbf{X}^{L T}=\mathbf{X}_{S L R}^{L T}-\mathbf{X}_{G N S S}^{L T}$

- same velocities for the LT stations

\section{Result}

$$
\delta \mathbf{X}=\left(\mathbf{X}_{S L R}^{\text {est }}-\mathbf{X}_{G N S S}^{\text {est }}\right)-\Delta \mathbf{X}^{L T}
$$


Results

\section{Local Ties}

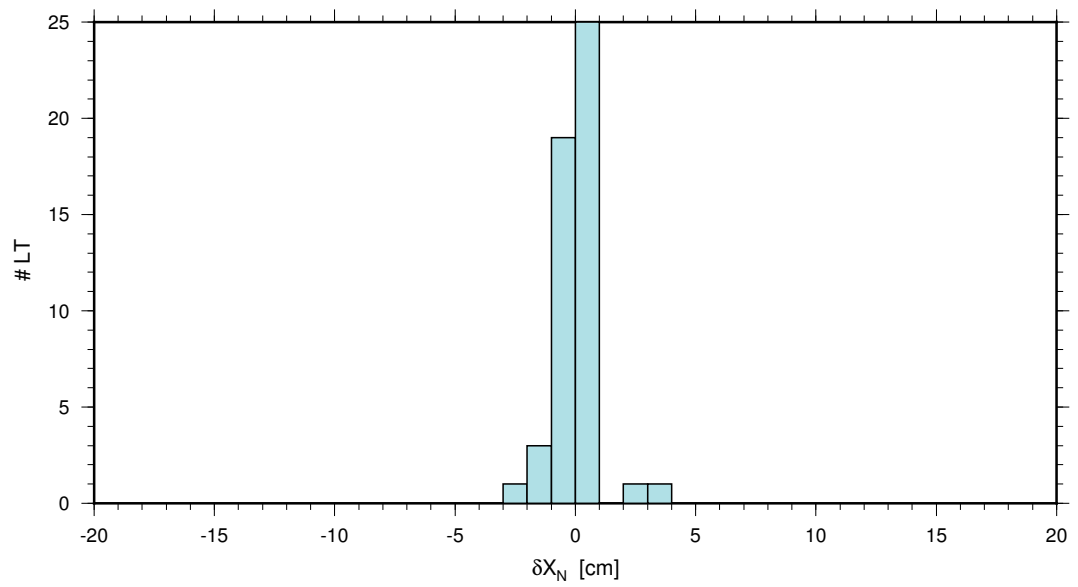

Figure: Histogram of differences $\delta X_{N}[\mathrm{~cm}]$ in the north component of estimated and measured local ties. 
Results

\section{Local Ties}

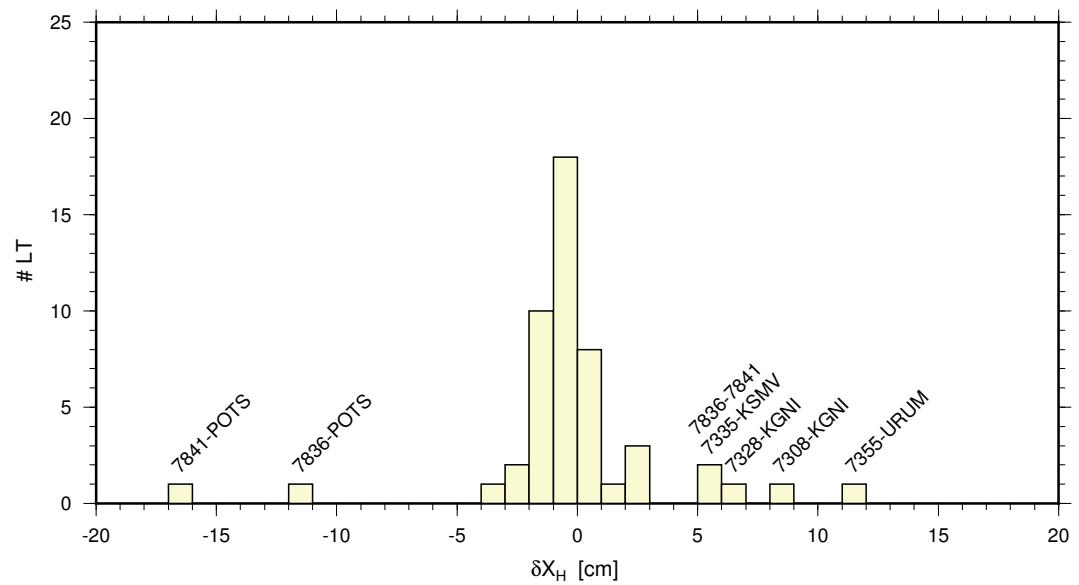

Figure: Histogram of differences $\delta X_{H}[\mathrm{~cm}]$ in the height component of estimated and measured local ties. 
Results

\section{Effect of the measured local ties}


Results

\section{Effect of the measured local ties}

Using a 14-parameter Helmert transformation

- between the single-technique solutions

- GNSS: $\mathbf{X}_{G N S S}^{\text {est }}$

- $\mathrm{SLR}+\mathrm{LT}: \mathbf{X}_{S L R}^{\text {est }}+\Delta \mathbf{X}^{L T}$ 
Transformation parameters of a 14-parameter Helmert transformation

\begin{tabular}{ll|cc}
\hline GNSS & & \multicolumn{2}{|c}{ SLR $+\mathrm{LT}$} \\
\hline$T_{X}[\mathrm{~mm}]$ & $\dot{T}_{X}[\mathrm{~mm} / \mathrm{a}]$ & 1,76 & $-0,38$ \\
$T_{Y}[\mathrm{~mm}]$ & $\dot{T}_{Y}[\mathrm{~mm} / \mathrm{a}]$ & $-16,51$ & 0,39 \\
$T_{Z}[\mathrm{~mm}]$ & $\dot{T}_{Z}[\mathrm{~mm} / \mathrm{a}]$ & 3,20 & $-0,14$ \\
$R_{X}[\mathrm{masec}]$ & $\dot{R}_{X}[\mathrm{masec} / \mathrm{a}]$ & 0,031 & $-0,012$ \\
$R_{Y}[\mathrm{masec}]$ & $\dot{R}_{Y}[\mathrm{masec} / \mathrm{a}]$ & 0,382 & $-0,007$ \\
$R_{Z}[\mathrm{masec}]$ & $\dot{R}_{Z}[\mathrm{masec} / \mathrm{a}]$ & $-0,147$ & 0,005 \\
$m[\mathrm{~mm} / \mathrm{km}]$ & $\dot{m}[\mathrm{~mm} / \mathrm{km} / \mathrm{a}]$ & $-0,00064$ & $-0,00025$ \\
\hline
\end{tabular}




\section{Outline}

\section{(1) Introduction}

\section{(2) Single-technique solutions}

\section{(3) Combined solution}

\section{(4) Conclusion}




\section{Summary}


Summary

- Combination of GNSS and SLR with minimum constraint conditions. 
Summary

- Combination of GNSS and SLR with minimum constraint conditions.

- Estimation of components of the local ties at co-located sites using the pole coordinates as global ties. 


\section{Summary}

- Combination of GNSS and SLR with minimum constraint conditions.

- Estimation of components of the local ties at co-located sites using the pole coordinates as global ties.

- Differences between estimated and measured local ties: 88\% in north, $52 \%$ in height component below $1 \mathrm{~cm}$. 


\section{Summary}

- Combination of GNSS and SLR with minimum constraint conditions.

- Estimation of components of the local ties at co-located sites using the pole coordinates as global ties.

- Differences between estimated and measured local ties: 88\% in north, $52 \%$ in height component below $1 \mathrm{~cm}$.

- Translation in direction of $Y$ and rotation of the network around $Y$ by using all measured local ties. 


\section{Summary}

- Combination of GNSS and SLR with minimum constraint conditions.

- Estimation of components of the local ties at co-located sites using the pole coordinates as global ties.

- Differences between estimated and measured local ties: $88 \%$ in north, $52 \%$ in height component below $1 \mathrm{~cm}$.

- Translation in direction of $Y$ and rotation of the network around $Y$ by using all measured local ties.

$\rightarrow$ Combination of different geodetic space techniques to realize a global terrestrial reference system in the framework of GGOS. 
Thank you very much for your attention.

\author{
susanne.glaser@tu-berlin.de
}

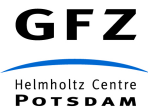

TECHNISCHE
UNIVERSITÄT
DRESDEN

Technische Universität 


\section{References}

Blewitt, G. (2003). Self-consistency in reference frames, geocenter definition, and surface loading of the solid Earth. J. Geophys. Res., 108(B2):2103.

Fritsche, M., Sośnica, K., Rodríguez-Solano, C., Steigenberger, P., Wang, K.,

Dietrich, R., Dach, R., Hugentobler, U., and Rothacher, M. (2014). Homogeneous reprocessing of GPS, GLONASS and SLR observations. J. Geod., pages 1-18.

IAG (2014). http://www.iag-ggos.org/about_geodesy/the_three_pillars.php.

Seitz, M., Angermann, D., Bloßfeld, M., Drewes, H., and Gerstl, M. (2012). The 2008 DGFI realization of the ITRS: DTRF2008. J. Geod., 86(12):1097-1123.

Thaller, D. (2008). Inter-technique combination based on homogeneous normal equation systems including station coordinates, Earth orientation and troposphere parameters. Deutsches GeoForschungsZentrum. Scientific Technical Report STR $08 / 15$. 


\section{Definition of core stations}

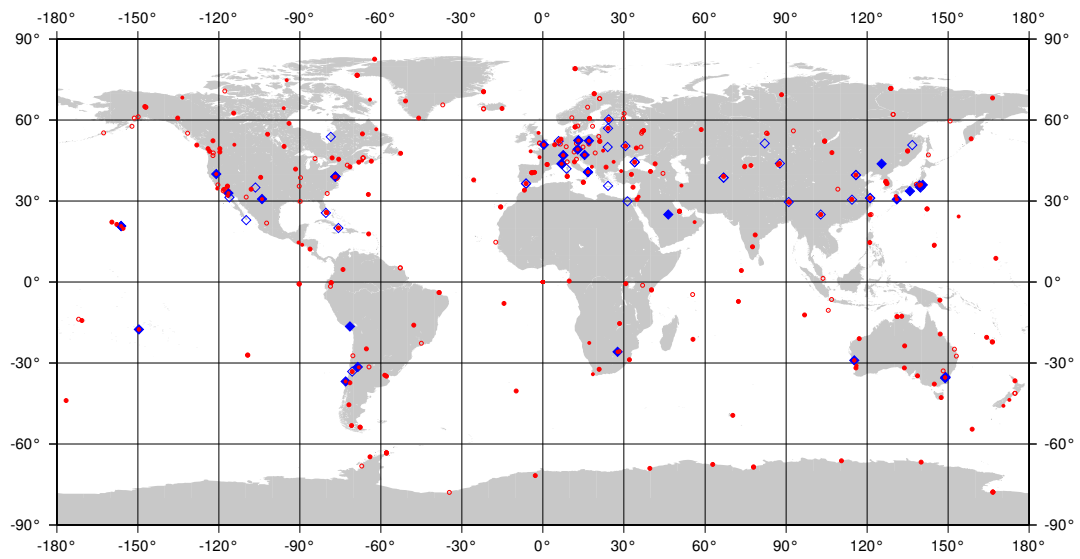

Figure: Definition of core stations (filled symbol) of the GNSS (red) the SLR (blue) network 

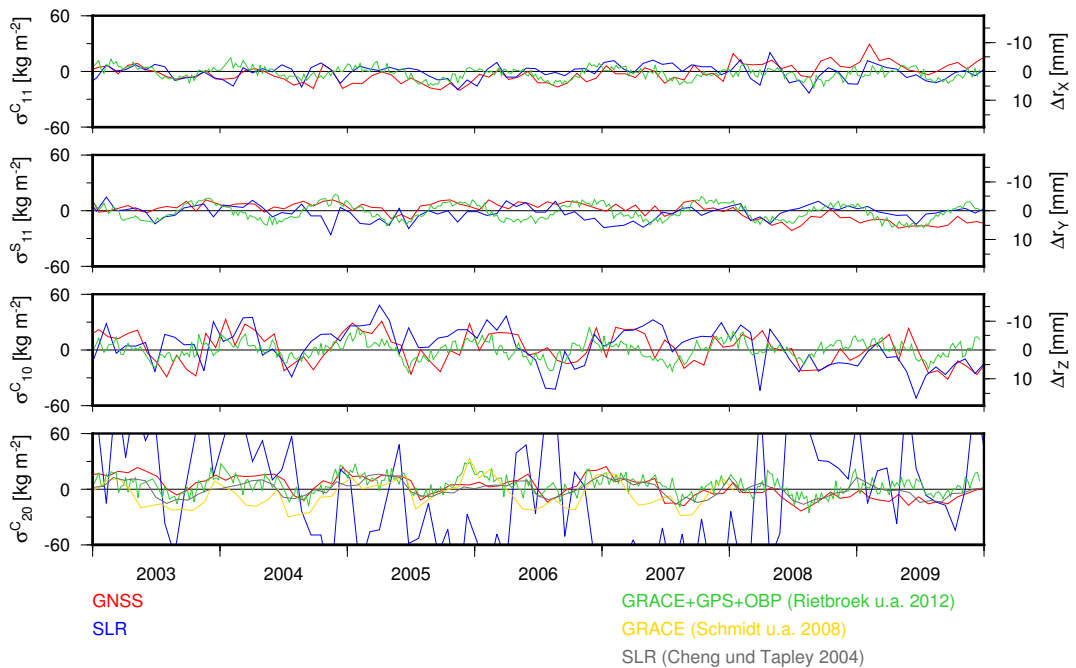

Figure: Surface load coefficients and difference of CF w. r. t. CM 


\section{Modeling of surface loads (Blewitt, 2003)}

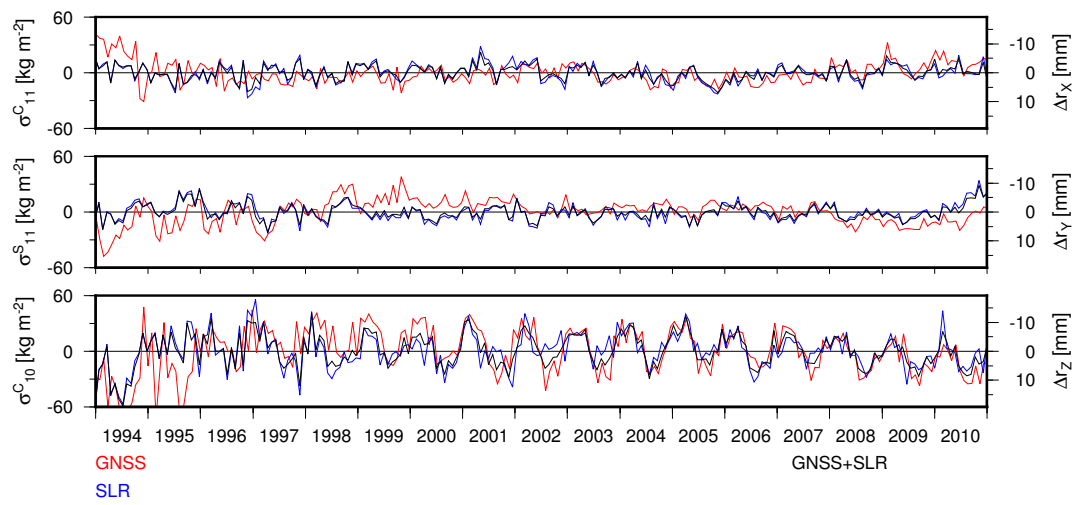

Figure: Degree- 1 surface load coefficients $\left(\sigma_{10}^{C}, \sigma_{11}^{C}, \sigma_{11}^{S}\right)$ (left) and differences $\left[\Delta \mathbf{r}_{\mathrm{CF}}\right]_{\mathrm{CM}}$ (right) 


\section{Modeling of surface loads (Blewitt, 2003)}

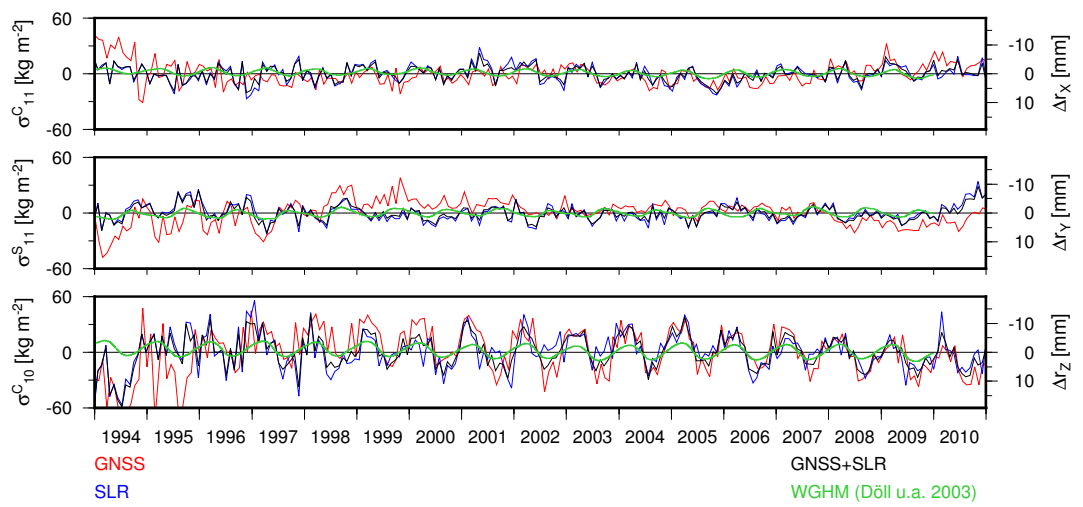

Figure: Degree- 1 surface load coefficients $\left(\sigma_{10}^{C}, \sigma_{11}^{C}, \sigma_{11}^{S}\right)$ (left) and differences $\left[\Delta \mathbf{r}_{\mathrm{CF}}\right]_{\mathrm{CM}}$ (right) 